\title{
Ocorrência de parasitóides de Spodoptera frugiperda (J. E. Smith)(Lep., Noctuidae) em lavouras de milho em Cachoeirinha, $\mathbf{R S}^{1}$
}

\author{
Occurrence of Spodoptera frugiperda (J. E. Smith) (lep. Noctuidae) parasitoids in maize crops \\ in Cachoeirinha, RS, Brazil
}

Sônia Thereza Bastos Dequech ${ }^{2}$ Rogério Fernando Pires da Silva ${ }^{3}$ Lidia Mariana Fiuza $^{4}$

\begin{abstract}
- NOTA -
RESUMO

O Rio Grande do Sul é um dos maiores produtores de milho do país, porém pouco se conhece em termos de inimigos naturais dos principais insetos-praga da cultura. O objetivo do presente trabalho foi realizar um levantamento dos parasitóides da lagarta-do-cartucho do milho, Spodoptera frugiperda. Para tanto, foram efetuadas amostragens em áreas de lavouras de milho do Instituto Riograndense do Arroz (IRGA), em Cachoeirinha, RS, nos anos agrícolas 1999/2000 e 2000/2001. A coleta dos insetos foi

parasitoids. In the first and second crop seasons, 1425 and 518 larvae were collected, respectively. Out of these, $18.11 \%$ and $22.01 \%$ were parasitized, with the following parasitoids being observed: Chelonus sp., Cotesia sp. and Exaticolus sp. (Hym., Braconidae), Campoletis flavicincta and Ophion sp. (Hym., Ichneumonidae), and Archytas incertus and Lespesia archippivora (Dip., Tachinidae). There was predominance of $\boldsymbol{C}$. flavicincta, corresponding to $63.57 \%$ and $76.32 \%$ of the parasitoids, respectively, for the first and second seasons. In C. flavicincta hiperparasitism occurred by Conura sp. (Chalcididae) and Aphanogmus sp. (Ceraphronidae).
\end{abstract} realizada semanalmente, durante o ciclo da cultura. As lagartas foram individualizadas e mantidas em laboratório até a emergência dos adultos ou dos parasitóides. Foram coletadas 1425 lagartas no primeiro período de amostragens e 518 no segundo. Destas, $18,11 \%$ e 22,01\% encontravam-se parasitadas, com a presença dos seguintes parasitóides: Chelonus sp., Cotesia sp. e Exaticolus sp. (Hym., Braconidae), Campoletis flavicincta e Ophion sp. (Hym., Ichneumonidae) e Archytas incertus e Lespesia archippivora (Dip., Tachinidae). Houve predomínio de C. flavicincta, correspondendo a $63,57 \%$ e 76,32\% dos parasitóides, respectivamente para o primeiro e segundo período de amostragens. Em C. flavicincta, ocorreu hiperparasitismo por Conura sp. (Chalcididae) $e$ Aphanogmus sp. (Ceraphronidae).

Palavras-chave: Campoletis flavicincta, Ichneumonidae Braconidae, Tachinidae, hiperparasitóides.

\section{ABSTRACT}

Rio Grande do Sul State is one of the greatest maize producers of the country, however, little is known in terms of the main natural enemies associated to insect pests in this crop. The objective of this work was to survey parasitoids of fall-armyworm, Spodoptera frugiperda. Samplings in maize areas of the Instituto Riograndense do Arroz (IRGA) were conducted in Cachoeirinha, $R S$, during the growth seasons of 1999/2000 and 2000/2001. Insect larvae were collected weekly, during the crop cycle. Larvae were individualized and kept in laboratory until emergence of adults or
Key words: Campoletis flavicincta, Ichneumonidae, Braconidae, Tachinidae, hiperparasitoids.

Spodoptera frugiperda (J. E. Smith, 1797) é um dos principais insetos-praga de gramíneas cultivadas, sendo responsável por altos níveis de desfolhamento. O método mais utilizado para o controle desta praga é o químico, mas, devido ao desequilíbrio no ecossistema, o controle biológico através do uso de parasitóides pode tornar-se uma alternativa viável (VALICENTE, 1989).

No Brasil, em diferentes regiões, os principais parasitóides correspondem às espécies: Campoletis flavicincta, Diadegma sp., Ophion flavidus (Ichneumonidae); Apanteles marginiventris, Chelonus sp. (Braconidae); Archytas incertus, Archytas marmoratus e Lespesia sp. (Tachinidae) (PATEL \& HABIB, 1984; VALICENTE, 1989; SILVA et al., 1997).

O presente trabalho teve como objetivo verificar a ocorrência de parasitóides que atuam como agentes de controle biológico natural de lagartas de $\boldsymbol{S}$. frugiperda em Cachoeirinha, RS.

\footnotetext{
${ }^{1}$ Parte da Tese de Doutorado do primeiro autor, apresentada ao Programa de Pós-graduação em Fitotecnia (Fitossanidade), Universidade Federal do Rio grande do Sul (UFRGS). Financiado com recursos da FAPERGS.

${ }^{2}$ Biólogo, Doutor, Professor Adjunto, Departamento de Defesa Fitossanitária, Universidade Federal de Santa Maria, 97105-900, Santa Maria, RS. E-mail: soniabd@terra.com.br.Autor para correspondência.

${ }^{3}$ Engenheiro Agrônomo, Doutor, Professor Adjunto, Departamento de Fitossanidade, UFRGS.

${ }^{4}$ Engenheiro Agrônomo, Doutora, Professora, Microbiologia, Centro 2, Universidade Vale do Rio dos Sinos.
} 
O levantamento de parasitóides foi realizado na Estação Experimental do IRGA, em Cachoeirinha, RS (2955'30' latitude sul e 5058'21" longitude oeste), nos anos agrícolas de 1999/2000 e 2000/2001, em áreas de cultivo de milho, circundadas por lavouras de arroz. No primeiro ano, foram utilizadas duas áreas, compreendendo o período de janeiro a abril de 2000 , com oito coletas na primeira área e seis na segunda. No segundo ano, as coletas, em número de seis, foram realizadas em janeiro e fevereiro de 2001, em apenas uma área. Em ambos os anos agrícolas, as áreas destinadas ao levantamento foram subdivididas em cinco blocos, cada bloco com 16 linhas de $8 \mathrm{~m}$. O espaçamento entre linhas era de $0,45 \mathrm{~m}$, sendo que cada linha apresentava, em média, 30 plantas.

A amostragem de lagartas foi realizada semanalmente, desde o início do ciclo da cultura até o final da ocorrência do inseto alvo. A cada coleta foi sorteada uma linha por bloco, sendo as lagartas capturadas e levadas ao Laboratório de Entomologia do Departamento de Fitossanidade da Faculdade de Agronomia da UFRGS. Em seguida, as lagartas foram individualizadas em tubos de ensaio, contendo dieta artificial, e mantidas em câmaras incubadoras tipo BOD (reguladas para $25^{\circ} \mathrm{C}$ e 12 horas de fotofase, e umidade relativa mantida próxima a $65 \%$ ) até a emergência dos adultos ou dos parasitóides. O número de lagartas parasitadas foi avaliado e os parasitóides obtidos foram enviados a especialistas para identificação.

Apesar do número total de insetos ter variado nos anos agrícolas estudados, observa-se que o percentual de parasitismo manteve-se próximo. Assim, de um total de 1425 lagartas coletadas em 2000, 258 $(18,11 \%)$ estavam parasitadas. Em 2001, o número de lagartas foi de 518, com 114 $(22,01 \%)$ parasitadas (Tabela 1). Estes valores foram semelhantes aos encontrados em distintos locais: 15,33\% em Ponta Grossa (PR) (LUCCHINI \& ALMEIDA, 1980); entre 9,16 e 36,11\% em municípios do Estado de São Paulo, no período de 1976 a 1980 (PATEL \& HABIB, 1984); 28,22\% em Espírito Santo do Pinhal (SP) (SILVEIRA et al., 1987); 23,79\% e 19,3\%, em diferentes regiões de Minas Gerais (VALICENTE, 1989; SILVA et al., 1997).

Para todas as espécies de parasitóides, houve a emergência de apenas um indivíduo por lagarta ou crisálida de $\boldsymbol{S}$. frugiperda. Verificou-se que houve diferença nas espécies coletadas em ambos os anos de amostragens, com os braconídeos Chelonus sp. e Cotesia sp. ocorrendo apenas no primeiro ano e o taquinídeo $\boldsymbol{L}$. archippivora no segundo. Porém, em ambas as épocas, houve predomínio dos himenópteros, correspondendo a 87,60\% e $86,84 \%$ do total de insetos coletados, respectivamente (Tabela 1). Nos anos agrícolas estudados, houve uma predominância de parasitóides larvais, com uma espécie ovo-larval (Chelonus sp.) e uma na qual a emergência ocorreu na fase pupal (A. incertus).

Considerando o número total de parasitóides encontrados, predominou C. flavicincta, correspondendo a cerca de $70 \%$ do total de parasitóides (Tabela 1), com maior emergência de fêmeas $(54,55 \%$ no primeiro ano e $59,02 \%$ no segundo). Este microimenóptero representou 56,67\% dos parasitóides de $S$. frugiperda obtidos em Ponta Grossa (PR) (LUCCHINI \& ALMEIDA, 1980) e 58,33\% em diferentes municípios do Estado de São Paulo (PATEL \& HABIB, 1984); em Cascavel (PR), Campoletis sp. correspondeu a 47,0\% dos parasitóides (VALICENTE \& BARRETO, 1999).

Tabela 1 - Parasitóides de Spodoptera frugiperda identificados a partir de amostragens realizadas na cultura do milho, nos anos agrícolas 1999/2000 e 2000/2001. EEA/IRGA - Cachoeirinha, RS.

\begin{tabular}{|c|c|c|c|c|c|}
\hline \multirow{3}{*}{ Parasitóide } & \multicolumn{4}{|c|}{ Ano agrícola } & \multirow{2}{*}{$\begin{array}{l}\text { Estágio de } \\
\text { emergência } \\
\end{array}$} \\
\hline & \multicolumn{2}{|c|}{$1999 / 2000$} & \multicolumn{2}{|c|}{$2000 / 2001$} & \\
\hline & $\mathrm{n}^{\circ}$ & $\%$ & $\mathrm{n}^{\mathrm{o}}$ & $\%$ & \\
\hline \multicolumn{6}{|l|}{ Hymenoptera } \\
\hline \multicolumn{6}{|l|}{ Braconidae } \\
\hline \multicolumn{6}{|l|}{ Cheloninae } \\
\hline Chelonus sp. & 40 & 15,50 & 0 & 0 & larval \\
\hline \multicolumn{6}{|l|}{ Homolabinae } \\
\hline Exaticolus sp. & 12 & 4,65 & 1 & 0,88 & larval \\
\hline \multicolumn{6}{|l|}{ Microgastrinae } \\
\hline Cotesia sp. & 4 & 1,55 & 0 & 0 & larval \\
\hline \multicolumn{6}{|l|}{ Ichneumonidae } \\
\hline \multicolumn{6}{|l|}{ Campopleginae } \\
\hline Campoletis flavicincta & 164 & 63,57 & 87 & 76,32 & larval \\
\hline \multicolumn{6}{|l|}{ Ophioninae } \\
\hline Ophion sp. & 6 & 2,33 & 11 & 9,65 & larval \\
\hline \multicolumn{6}{|l|}{ Diptera } \\
\hline \multicolumn{6}{|l|}{ Tachinidae } \\
\hline \multicolumn{6}{|l|}{ Goniinae } \\
\hline Lespesia archippivora & 0 & 0 & 6 & 5,26 & larval \\
\hline \multicolumn{6}{|l|}{ Tachininae } \\
\hline Archytas incertus & 32 & 12,40 & 9 & 7,89 & pupal \\
\hline Total & 258 & 100 & 114 & 100 & \\
\hline
\end{tabular}


Quando foram encontrados casulos de $\boldsymbol{C}$. flavicincta, os mesmos foram coletados e considerados no número total de $S$. frugiperda sendo, posteriormente, catalogados dentre os insetos parasitados por $\boldsymbol{C}$. flavicincta. Este total correspondeu a 46 no ano agrícola 1999/2000 e seis em 2000/2001. Foi observada a ocorrência de hiperparasitóides em cinco casulos coletados no primeiro ano. De quatro desses casulos emergiram Conura sp. (Chalcididae). No casulo restante, foi verificada a emergência de 15 indivíduos de Aphanogmus sp. (Ceraphronidae).

Conura sp. é citado como hiperparasitóide de Aleiodes stigmator, Cotesia sp., Cotesia orobenae e Cotesia glomerata (FRANA\& O'NEIL, 1993; GAINES \& KOK, 1995; DOMINGUEZ et al., 1999; GAINES \& KOK, 1999). PATEL \& HABIB (1984) encontraram os seguintes hiperparasitóides de $\boldsymbol{C}$. flavicincta no Estado de São Paulo: Spilochalcis chapadae, Ceraphron (Caliceras) sp. e um Hemitelini (Ichneumonidae) não identificado. O gênero Spilochalcis é considerado sinonímia de Conura por GAINES \& KOK (1995). Com relação a Aphanogmus sp., foi referido hiperparasitando Cotesia sp. (PETER \& DAVID, 1993; KFIR, 1995), não havendo citação de sua ocorrência em $\boldsymbol{C}$. flavicincta.

Estudos complementares devem ser realizados visando avaliar as espécies de parasitóides encontradas, com vistas à possível utilização como agentes de controle biológico de S. frugiperda, em especial $\boldsymbol{C}$. flavicincta, por ser predominante na região.

\section{AGRADECIMENTOS}

Os autores agradecem ao Instituto Riograndense do Arroz (IRGA) pela cedência e manutenção das áreas de cultivo de milho utilizadas nos experimentos. Também aos pesquisadores Dr. Virendra Gupta, Entomology \& Nematology Dept., University of Florida, Gainesville, FL, pela identificação de Campoletis flavicincta; Dr. Ronaldo Toma, Museu de Entomologia, USP por ter identificado os taquinídeos; e Dr. Gerard Delvare, do CIRAD, Montpellier, França e Dr. Ayres Menezes Jr. da Universidade Estadual de Londrina, PR pela identificação dos demais parasitóides e hiperparasitóides.

\section{REFERÊNCIAS BIBLIOGRÁFICAS}

DOMINGUEZ, O. et al. Parasitoides de lepidopteros defoliadores del platano (Musa AAB, cv. Harton) en el sur del
Lago de Maracaibo, Venezuela. Revista de la Facultad de Agronomia, v.16, p.95-101, 1999.

FRANA, J.E.; O'NEIL, R.J. Parasitism of late instar larvae of the cattail caterpillar, Simyra henrici (Grote) (Lepidoptera: Noctuidae) in Indiana. Journal of the Kansas Entomological Society, v.66, p.399-404, 1993.

GAINES, D.N.; KOK, L.T. Cotesia orobenae (Hymenoptera: Braconidae), a gregarious endoparasitoid of Evergestis rimosalis (Lepidoptera:Pyralidae), and hyperparasitoids in Virginia Brassica crops. Biological Control, v.5, p.573-580, 1995.

GAINES, D.N.; KOK, L.T. Impact of hyperparasitoids on Cotesia glomerata in southwestern Virginia. Biological Control, v.14, p.19-28, 1999.

KFIR, R. Parasitoids of the African stem borer, Busseola fusca (Lepidoptera: Noctuidae), in South Africa. Bulletin of Entomological Research, v.85, p.369-377, 1995.

LUCCHINI, F.; ALMEIDA, A.A. Parasitas da Spodoptera frugiperda (Smith \& Abbot, 1797) (Lep., Noctuidae), lagarta do cartucho do milho, encontrado em Ponta Grossa, Pr. Anais da Sociedade Entomológica do Brasil, v.9, p.115$121,1980$.

PATEL, P.N.; HABIB, M.E.M. Levantamento e eficiência de insetos parasitos de Spodoptera frugiperda (Abbot \& Smith, 1797) (Lepidoptera, Noctuidae). Revista de Agricultura, v.59, p.229237, 1984.

PETER, C.; DAVID, B.V. Studies on the hiperparasitoids of Diaphania indica (Lepidoptera: Pyralidae) through Apanteles taragamae (Hymenoptera: Braconidae). Journal of the Bombay Natural History Society, v.90, p.412-416, 1993.

SILVA, F.M.A.; FOWLER, H.G.; LEMOS, R.N.S. Parasitismo em lagarta-do-cartucho, Spodoptera frugiperda (Smith) na região do Triângulo Mineiro, MG.. Anais da Sociedade Entomológica do Brasil, v.26, p.235-241, 1997.

SILVEIRA, J.C.F. da et al. Ocorrência de parasitóides de Spodoptera frugiperda (J.E. Smith, 1797) em cultura de mi1ho, em Espírito Santo do Pinhal-SP. Ecossistema, v.12, p.4144, 1987.

VALICENTE, F.H. Levantamento dos inimigos naturais de Spodoptera frugiperda (J. E. Smith, 1797) (Lepidoptera, Noctuidae), em diferentes regiões do Estado de Minas Gerais. Anais da Sociedade Entomológica do Brasil, v.18, p.119-130, 1989.

VALICENTE, F.H.; BARRETO, M.R. Levantamento dos inimigos naturais da lagarta do cartucho do milho, Spodoptera frugiperda (J. E. Smith) (Lepidoptera: Noctuidae), na região de Cascavel, PR. Anais da Sociedade Entomológica do Brasil, v.28, p.333-337, 1999. 